\title{
Caracterização florística e fitossociológica em área de Caatinga para fins de manejo florestal no município de São Francisco-PI
}

\author{
Alexandro Dias Martins Vasconcelos ${ }^{1 *}$, Ikallo George Nunes Henriques ${ }^{1}$, Maílson Pereira de \\ Souza $^{1}$, William de Sousa Santos ${ }^{1}$, Wellington de Sousa Santos ${ }^{1}$, Gabriela Gomes Ramos ${ }^{1}$
}

\begin{abstract}
RESUMO: Objetivou-se neste trabalho conhecer a diversidade florística-fitossociológica e a produção madeireira de uma área de caatinga destinada ao Manejo Florestal Sustentável. Realizou-se o inventário florestal por meio da Amostragem Aleatória Simples, instalando-se 19 unidades amostrais de 20 x $20 \mathrm{~m}$. Avaliou-se os parâmetros fitossociológicos da estrutura horizontal e vertical, a diversidade florística das espécies e a produção madeireira da área. As famílias Fabaceae, Apocynaceae e Combretaceae foram as mais representativas. As três espécies de maior representação na área foram Combretum glaucocarpum Mart., Xylopia sericea A.St.-Hil. e Terminalia fagifolia Mart . $\mathrm{O}$ índice de diversidade de Shannon-Weaver (H') observado para a área foi de 3,08 nats. ind ${ }^{-1}$. A área basal estimada para a área foi de 3,08 $\mathrm{m}^{2} \cdot \mathrm{ha}^{-1}$. O volume real estimado foi de $112,9 \mathrm{~m}^{3} \cdot \mathrm{ha}^{-1}$. Para todo o efeito de comparação, a diversidade de espécies no fragmento estudado foi considerada elevada para o padrão normal em áreas de Caatinga, haja vista que quando comparada com outros estudos, apresentou superior.
\end{abstract}

Palavras-chave: estrutura de comunidades, Caatinga, produtividade madeireira.

\section{Floristic and phytosociological characterization in the Caatinga area for forest management purposes in the municipality of São Francisco-PI.}

\begin{abstract}
The objective of this work was to know the floristic-phytosociological diversity and the timber production of a caatinga area destined to the Sustainable Forest Management. The forest inventory was carried out by means of Simple Random Sampling, installing 19 sample units of 20 x $20 \mathrm{~m}$. The phytosociological parameters of the horizontal and vertical structure, the floristic diversity of the species and the timber production of the area were evaluated. The families Fabaceae, Apocynaceae and Combretaceae were the most representative. The three most representative species in the area were Combretum glaucocarpum Mart., Xylopia sericea A.St.-Hil. and Terminalia fagifolia Mart. The diversity index of Shannon-Weaver $\left(\mathrm{H}^{\prime}\right)$ observed for the area was 3.08 nats. $^{-1}$. The estimated basal area for the area was $3.08 \mathrm{~m}^{2} \cdot \mathrm{ha}^{-1}$. The estimated real volume was $112.9 \mathrm{~m}^{3} \cdot \mathrm{ha}^{-1}$. For all the comparison effect, the species diversity in the studied fragment was considered high for the normal pattern in Caatinga areas, since when compared with other studies, it was superior.
\end{abstract}

Keywords: community structure, Caatinga, timber productivity.

\section{INTRODUÇÃO}

O Bioma Caatinga possui aproximadamente cerca de $900.000 \mathrm{~km} 2$, representando quase $54 \%$ do nordeste brasileiro, bem como $11 \%$ do território nacional. A Caatinga abrange os nove estados nordestinos (Ceará, Bahia, Rio Grande do Norte, Paraíba, Pernambuco, Alagoas, Sergipe, sudoeste do Piauí e do Maranhão, e norte de Minas Gerais (ANDRADE et al., 2005).

A Caatinga apresenta características peculiares como fisionomia xerófila e florística variada, com grande capacidade adaptativa, elevado grau de endemismo consistindo em um dos tipos de vegetação mais difícil de ser definido, devido sua heterogeneidade relacionada à sua fisionomia e sua composição florística (RODAL et. al.,1992; PRADO, 2003; DIAS e KIILL, 2008).
Com características únicas, a Caatinga é composta por espécies herbáceas, cactáceas, bromeliáceas, arbustivas e arbóreas de pequeno porte, em maior parte providas de espinhos ou acúleos, apresentando caducifólia, perdendo suas folhas no início da estação seca (DRUMOND et al., 2000).

Com intenso processo de degradação, a Caatinga vem passando por inúmeros problemas relacionados ao uso inadequado dos seus recursos naturais. $\mathrm{O}$ desmatamento e as queimadas são os principais fatores degradantes do bioma. Na maioria das vezes os recursos naturais são explorados de forma insustentável, o que provoca a aceleração dos processos de degradação e intensificam a desertificação no semiárido, provocando a extinção 
de algumas espécies vegetais (FERNANDES e MEDEIROS, 2009; GARIGLIO, 2010; CALIXTO JÚNIOR; DRUMOND, 2014).

Há muito tempo a ação antrópica é responsável pela degradação da Caatinga, combinado a isto, ressalta-se o pouco entendimento sobre o bioma, sobre tudo em relação de como utilizar os recursos oferecidos pela mesma, uma vez que a Caatinga possui aspectos particulares que devem ser considerados, para que a mesma possa de regenerar e assim, novamente ser explorada.

Analisar a composição florística e fitossociológica em florestas secas como a caatinga estabelece uma concepção detalhada da diversidade de espécies vegetais, e de como elas estão distribuídas em determinado ambiente, por meio da análise estrutural que permite a estimativa da distribuição de indivíduos por espécies e consequentemente por famílias (SOUZA, 2009).

Nesse contexto o entendimento sobre a florística e fitossociologia é de suma importância, visto que, representa os passos iniciais para o conhecimento ecológico dessa vegetação, outro sim, é que estabelece graus de dominância entre as espécies estudadas. Desta forma conhecer a estrutura e dinâmica de uma floresta, termina contribuindo e gerando subsídios para a conservação dos recursos naturais, bem como para a conservação de áreas similares (CHAVES et al., 2013; BULHÕES et al., 2015).

Dessa forma, objetivou-se realizar um estudo florístico e fitossociológico do componente florestal em um fragmento de Caatinga e a produção madeireira de uma área de caatinga destinada ao Manejo Florestal Sustentável, localizado na Unidade Produtiva Familiar Umbaúba II, no município de São Francisco do Piauí.

\section{MATERIAL E MÉTODOS}

O município de São Francisco está localizado na microrregião de Floriano, compreendendo uma área irregular de $1.422 \mathrm{~km}^{2}$. A sede municipal tem as coordenadas geográficas de $07^{\circ} 15^{\prime} 05^{\prime}$ ' de latitude sul e 42 $32^{\prime} 36^{\prime \prime}$ de longitude oeste de Greenwich e dista cerca de $317 \mathrm{~km}$ de Teresina. As condições climáticas do município de São Francisco do Piauí (com altitude da sede a $158 \mathrm{~m}$ acima do nível do mar) apresentam temperaturas mínimas de $25^{\circ} \mathrm{C}$ e máximas de $39^{\circ} \mathrm{C}$, com clima semi úmido e quente. A precipitação pluviométrica média anual (registrada, na sede do município, $800 \mathrm{~mm}$ ) é definida com isoietas anuais entre 800 a $1.400 \mathrm{~mm}$ e trimestres janeiro-fevereiro-março e dezembro janeiro-fevereiro como os mais chuvosos (AGUIAR; GOMES, 2004).

Os solos da região são provenientes da alteração de arenitos, siltito, folhelho, calcário e laterito.
Compreendem solos litólicos, álicos e distróficos, de textura média, pouco desenvolvidos, rasos a muito rasos.

A vegetação natural que ocorre na UPF Umbauba II é do tipo floresta caducifólia e/ou floresta subcaducifólia/cerrado se constituindo de caatinga arbórea densa. $\mathrm{O}$ fragmento inventariado situa-se numa área de transição, possuindo tanto espécies de cerrado quanto de caatinga. Devido sua heterogeneidade espaço-ambiental, sua cobertura vegetal apresenta uma mistura florística, onde coexistem espécies dos biomas Cerrado e Caatinga.

\section{Coleta e tratamento dos dados}

Para a realização do levantamento fitossociológico foi considerada uma área com aproximadamente 250 ha de mata nativa, onde foram alocadas de forma aleatória 19 parcelas com tamanho de $20 \times 20 \mathrm{~m}\left(400 \mathrm{~m}^{2}\right)$, resultando numa área inventariada de $7.600 \mathrm{~m}^{2}$.

Mediu-se dentro das 19 parcelas as espécies florestais de composição arbóreo-arbustiva com circunferência a altura do peito (CAP) igual ou superior a $10 \mathrm{~cm}$. As mesmas foram identificadas in loco através dos aspectos dendrológico e, quando possível, realizada a coleta do material botânico para posterior identificação e/ou confirmação dos nomes científicos por consultas à literatura especializada (LORENZI, 2002; SAMPAIO et al., 2005; GAMARRA- ROJAS et al., 2010; SIQUEIRA FILHO et al., 2009; FORZZA et al., 2012; SILVA et al., 2010; LIMA, 2012; MAIA, 2012). Para classificação das espécies encontradas foi adotado o sistema APG II (2003), sendo classificadas até o nível de espécie.

Em posse dos dados foram avaliados os parâmetros fitossociológicos da estrutura horizontal e vertical das espécies amostradas (FELFILI e REZENDE, 2003), a diversidade florística de espécies através do Índice de Shannon-Weaver (H'), dominância de Simpson (C) e equabilidade de Pielou $\left(\mathrm{J}^{\prime}\right)$.

Quanto ao rendimento volumétrico foi calculado considerando o DAP a 1,30 m com casca das árvores e arbustos mensurados nas unidades de amostras das árvores adultas, com a aplicação das expressões:

$$
\mathrm{VCc} / \mathrm{c}=\frac{\pi *\left(\mathrm{DAP}^{2}\right) * \mathrm{Ht}}{40000}
$$

Em que: $\mathbf{V C c} / \mathbf{c}=$ volume cilíndrico da árvore com casca; $\boldsymbol{\pi}=$ "pi" $(3,1416 \ldots) ; \mathbf{D A P} \mathbf{P}^{2}=$ diâmetro a altura do peito, ao quadrado (em centímetros); $\mathbf{H t}=$ altura total da árvore (em metros); $\mathbf{4 0 . 0 0 0}=$ fator de conversão quadrática de centímetros para metros (do DAP);

$$
V R=\mathrm{VC} * \mathrm{ff}
$$


Em que: VR = volume real (em metros cúbicos $\left.\mathrm{m}^{3}\right) ; \mathbf{f f}=$ fator de forma $(0,9$ adimensional) (ZAKIA, PAREYN e RIEGELHAUPT, 1988).

Para a estimativa dos volumes empilhados, foi aplicada a seguinte fórmula:

$$
V E=\mathrm{VR} * \mathrm{fe}
$$

Em que: VE = volume empilhado (em estéreos); VR $=$ volume real (metro cúbico); fe $=$ fator de empilhamento (3,41 adimensional) (ZAKIA, PAREYN e RIEGELHAUPT, 1988).

\section{RESULTADOS E DISCUSSÃO}

Tabela 1. Relação das espécies identificadas encontrados nas parcelas amostrais na área da UPF Umbaúba II

\begin{tabular}{|c|c|c|}
\hline Família/Espécies & Nome vulgar & Hábito \\
\hline \multicolumn{3}{|l|}{ Anacardiaceae } \\
\hline Lithraea molleoides (Vell.) Engl. & Aroeira branca & Árbóreo \\
\hline \multicolumn{3}{|l|}{ Annonaceae } \\
\hline Annona leptopetala (R.E.Fr.) H.Rainer. & Rama de Bezerro & Arbustivo \\
\hline Annona leptopetala (R.E.Fr.) H.Rainer & Bananinha & Árbóreo \\
\hline Xylopia sericea A.St.-Hil. & Pindaíba & Árbóreo \\
\hline Apocynaceae & & Arbustivo \\
\hline Aspidosperma parvifolium A.DC. & Amarelo & Árbóreo \\
\hline Aspidosperma multiflorum A.DC. & Pequiá & Árbóreo \\
\hline Aspidosperma pyrifolium Mart. & Pereiro & Árbóreo \\
\hline Aspidosperma cuspa (Kunth) S.F.Blake ex Pittier & Pereiro branco & Arbustivo \\
\hline \multicolumn{3}{|l|}{ Asteraceae } \\
\hline Gochnatia polymorpha (Less.) Cabrera & Candeia & Árbóreo \\
\hline Astronium fraxinifolium Schott & Gonçalo Alves & Árbóreo \\
\hline \multicolumn{3}{|l|}{ Bignoniaceae } \\
\hline Handroanthus impetiginosus (Mart. ex DC.) Mattos & Pau d'arco & Árbóreo \\
\hline Jacaranda jasminoides (Thunb.) Sandwith & Chifre de carneiro & Árbóreo \\
\hline \multicolumn{3}{|l|}{ Combretaceae } \\
\hline Combretum duarteanum Cambess. & Jacaré & Árbóreo \\
\hline Terminalia fagifolia Mart. & Chapada & Árbóreo \\
\hline Combretum leprosum Mart. & Mofumbo & Árbustivo \\
\hline Combretum glaucocarpum Mart. & Sipaúba & Árbóreo \\
\hline \multicolumn{3}{|l|}{ Dilleniaceae } \\
\hline Curatella americana $\mathrm{L}$. & Sambaíba & Árbóreo \\
\hline \multicolumn{3}{|l|}{ Euphorbiaceae } \\
\hline Croton blanchetianus Baill. & Marmeleiro & Árbóreo \\
\hline \multicolumn{3}{|l|}{ Fabaceae } \\
\hline Machaerium acutifolium Vogel & Violete & Árbóreo \\
\hline Poincianella pyramidalis (Tul.) L.P.Queiroz & Catingueira & Árbóreo \\
\hline Bauhinia cheilantha (Bong.) Steud. & Mororó & Árbóreo \\
\hline Dalbergia brasiliensis Vogel & Jacarandá & Árbóreo \\
\hline Senna acuruensis (Benth.) H.S.Irwin \& Barneby & Canela de Velho & Árbóreo \\
\hline Pterocarpus Jacq. & Pau Sangue & Abóreo \\
\hline Mimosa caesalpiniifolia Benth. & Sabiá & Arbóreo \\
\hline Dimorphandra mollis & Fava danta & Arbóreo \\
\hline Andira fraxinifolia Benth. & Jacarandá & Arbóreo \\
\hline Mimosa tenuiflora (Willd.) Poir. & Jurema preta & Arbóreo \\
\hline Vatairea macrocarpa (Benth.) Ducke & Amargoso & Arbóreo \\
\hline Mimosa acutistipula (Mart.) Benth. & Jurema vermelha & Arbóreo \\
\hline Parkia pendula (Willd.) Benth. ex Walp. & Faveira & Arbóreo \\
\hline Copaifera duckei Dwyer & Podói & Arbóreo \\
\hline Hymenaea courbaril L. & Jatobá & Arbóreo \\
\hline \multicolumn{3}{|l|}{ Malvaceae } \\
\hline Luehea candicans Mart. & Açoita cavalo & Arbóreo \\
\hline \multicolumn{3}{|l|}{ Moraceae } \\
\hline Brosimum sp. & Inharé & Arbóreo \\
\hline
\end{tabular}




\begin{tabular}{lcc}
\hline \multicolumn{1}{c}{ Família/Espécies } & Nome vulgar & Hábito \\
\hline Nyctaginaceae & & Arbóreo \\
Pisonia sp & Pau Piranha & \\
& & \\
Myrtaceae & & Arbóreo \\
Myrcia tomentosa (Aubl.) DC. & Goiaba brava & Arbóreo \\
Eugenia dysenterica DC. & Cagaita & \\
Malpighiaceae & & Arbóreo \\
Byrsonima verbascifolia (L.) DC. & Muricí & Arbóreo \\
Diplopterys A.Juss. & Birro preto & \\
Vochysiaceae & & \\
Callisthene fasciculata Mart. & Arbóreo \\
Qualea grandiflora Mart. & Pau de terra & Arbóreo \\
Inderterminada/desconhecida & & \\
Inderterminada & Tingui & Arbóreo \\
Inderterminada & Tapa quintal & Arbóreo \\
Inderterminada & Arbóreo \\
Inderterminada & Quebra machado & Arbóreo \\
Inderterminada & Totó & Arbóreo \\
Desconhecida & desconhecida & Arbóreo \\
\hline
\end{tabular}

Souza et al. (2017), em estudo num fragmento de ecótono vegetacional no sul do Piauí, verificaram o predomínio de espécies pertencentes às famílias Fabaceae e Combretaceae. Observa-se que há presença de espécies proeminentemente nas listagens, salienta-se que embora distantes geograficamente as duas listas apresentam espécies ocorrentes em ambientes transicionais a citar: Copaifera lagsdorfii (Podói), Myrcia tomentosa (Goiaba brava) e Aspidosperma pyrifolium (Pequiá).

As três espécies mais abundantes foram Combretum glaucocarpum Mart. (135), Xylopia sericea A.St.-Hil. (120), Terminalia fagifolia Mart.
Outras espécies de grande destaque foram; Aspidosperma discolor A.DC.(64) e Caryocar sp. (61). Com relação ao valor de importância (IVI), que indica o quanto é ecologicamente importante uma determinada espécie na comunidade, constituído pela soma de todos os valores dos parâmetros relativos, a espécie Terminalia fagifolia Mart foi a que apresentou maior IVI de $12,12 \%$, seguida da Combretum glaucocarpum Mart. com 8,78\%, Xylopia sericea A.St.-Hil. Com 8,46\%, Aspidosperma discolor A.D.C. com 7,97\% e $\begin{array}{llll}\text { Caryocar } & \text { sp. } & \text { com } & \end{array}$ (70).

Tabela 2. Relação das espécies inventariadas, na UPF Umbaúba II, município de São Francisco, PI. Os valores estão em ordem decrescente de IVI. Em que N= número de indivíduos, FA= Frequência Absoluta (\%), FR= Frequência Relativa (\%), DA= Densidade Absoluta (ind.ha $\left.{ }^{-1}\right), \mathrm{DR}=$ Densidade Relativa (\%), DoA= Dominância Absoluta $\left(\mathrm{m}^{2} \cdot \mathrm{ha}^{-1}\right)$, DoR= Dominância Relativa $(\%)$, IVI= Índice de Valor de Importância (\%) IVC $=$ Índice de Valor de Cobertura $(\%)$

\begin{tabular}{lcccccccc}
\multicolumn{1}{c}{ Espécies } & NI & DA & DR & FA & FR & DoA & DoR & IVI (\%) \\
\hline Terminalia fagifolia Mart. & 70 & 92,105 & 7,4 & 94,74 & 6,19 & 3,922 & 22,78 & 12,12 \\
Combretum glaucocarpum Mart. & 135 & 177,632 & 14,27 & 89,47 & 5,84 & 1,074 & 6,24 & 8,78 \\
Xylopia sericea A.St.-Hil. & 120 & 157,895 & 12,68 & 94,74 & 6,19 & 1,119 & 6,5 & 8,46 \\
Aspidosperma discolor A.DC. & 64 & 84,211 & 6,77 & 68,42 & 4,47 & 2,184 & 12,68 & 7,97 \\
Caryocar sp. & 61 & 80,263 & 6,45 & 89,47 & 5,84 & 0,898 & 5,21 & 5,83 \\
Combretum duarteanum Cambess. & 55 & 72,368 & 5,81 & 57,89 & 3,78 & 0,879 & 5,11 & 4,9 \\
Annona leptopetala (R.E.Fr.) H.Rainer & 44 & 57,895 & 4,65 & 84,21 & 5,5 & 0,686 & 3,99 & 4,71 \\
Myrcia tomentosa (Aubl.) DC. & 54 & 71,053 & 5,71 & 63,16 & 4,12 & 0,599 & 3,48 & 4,44 \\
Machaerium acutifolium Vogel & 35 & 46,053 & 3,7 & 68,42 & 4,47 & 0,741 & 4,31 & 4,16 \\
Inderterminada & 28 & 36,842 & 2,96 & 68,42 & 4,47 & 0,637 & 3,7 & 3,71 \\
Poincianella pyramidalis (Tul.) L.P.Queiroz & 37 & 48,684 & 3,91 & 42,11 & 2,75 & 0,304 & 1,76 & 2,81 \\
Gochnatia polymorpha (Less.) Cabrera & 16 & 21,053 & 1,69 & 52,63 & 3,44 & 0,511 & 2,97 & 2,7 \\
Xylopia aromatica (Lam.) Mart. & 24 & 31,579 & 2,54 & 63,16 & 4,12 & 0,149 & 0,87 & 2,51 \\
Eugenia dysenterica DC. & 19 & 25 & 2,01 & 52,63 & 3,44 & 0,234 & 1,36 & 2,27 \\
Bauhinia cheilantha (Bong.) Steud. & 29 & 38,158 & 3,07 & 36,84 & 2,41 & 0,08 & 0,46 & 1,98 \\
Dalbergia brasiliensis Vogel & 6 & 7,895 & 0,63 & 31,58 & 2,06 & 0,449 & 2,61 & 1,77 \\
Aspidosperma parvifolium A.DC. & 5 & 6,579 & 0,53 & 26,32 & 1,72 & 0,508 & 2,95 & 1,73 \\
Pterocarpus Jacq. & 7 & 9,211 & 0,74 & 26,32 & 1,72 & 0,379 & 2,2 & 1,55 \\
Desconhecida 1 & 13 & 17,105 & 1,37 & 36,84 & 2,41 & 0,062 & 0,36 & 1,38 \\
Combretum leprosum Mart. & 14 & 18,421 & 1,48 & 36,84 & 2,41 & 0,031 & 0,18 & 1,36 \\
Mimosa caesalpiniifolia Benth. & 14 & 18,421 & 1,48 & 10,53 & 0,69 & 0,221 & 1,29 & 1,15 \\
Brosimum Sw. & 6 & 7,895 & 0,63 & 21,05 & 1,37 & 0,22 & 1,28 & 1,1 \\
Qualea grandiflora Mart. & 11 & 14,474 & 1,16 & 21,05 & 1,37 & 0,111 & 0,65 & 1,06 \\
Byrsonima verbascifolia (L.) DC. & 7 & 9,211 & 0,74 & 31,58 & 2,06 & 0,056 & 0,33 & 1,04 \\
Inderterminada & 8 & 10,526 & 0,85 & 31,58 & 2,06 & 0,038 & 0,22 & 1,04 \\
\hline & & & & & & &
\end{tabular}




\begin{tabular}{|c|c|c|c|c|c|c|c|c|}
\hline Espécies & NI & DA & DR & FA & FR & DoA & DoR & IVI (\%) \\
\hline Astronium fraxinifolium Schott & 7 & 9,211 & 0,74 & 21,05 & 1,37 & 0,144 & 0,84 & 0,98 \\
\hline Inderterminada & 3 & 3,947 & 0,32 & 15,79 & 1,03 & 0,208 & 1,21 & 0,85 \\
\hline Dimorphandra mollis & 5 & 6,579 & 0,53 & 21,05 & 1,37 & 0,11 & 0,64 & 0,85 \\
\hline Hymenaea courbaril L. & 5 & 6,579 & 0,53 & 15,79 & 1,03 & 0,12 & 0,7 & 0,75 \\
\hline Luehea candicans Mart. & 6 & 7,895 & 0,63 & 21,05 & 1,37 & 0,041 & 0,24 & 0,75 \\
\hline Lithraea molleoides (Vell.) Engl. & 2 & 2,632 & 0,21 & 10,53 & 0,69 & 0,125 & 0,73 & 0,54 \\
\hline Vatairea macrocarpa (Benth.) Ducke & 3 & 3,947 & 0,32 & 15,79 & 1,03 & 0,037 & 0,21 & 0,52 \\
\hline Handroanthus impetiginosus (Mart. ex DC.) Mattos & 5 & 6,579 & 0,53 & 10,53 & 0,69 & 0,044 & 0,25 & 0,49 \\
\hline Mimosa tenuiflora (Willd.) Poir. & 7 & 9,211 & 0,74 & 5,26 & 0,34 & 0,051 & 0,3 & 0,46 \\
\hline Copaifera martii Hayne & 2 & 2,632 & 0,21 & 10,53 & 0,69 & 0,037 & 0,21 & 0,37 \\
\hline Jacaranda jasminoides (Thunb.) Sandwith & 2 & 2,632 & 0,21 & 10,53 & 0,69 & 0,015 & 0,09 & 0,33 \\
\hline Mimosa acutistipula (Mart.) Benth. & 2 & 2,632 & 0,21 & 10,53 & 0,69 & 0,004 & 0,02 & 0,31 \\
\hline Desconhecida 2 & 1 & 1,316 & 0,11 & 5,26 & 0,34 & 0,056 & 0,32 & 0,26 \\
\hline Callisthene fasciculata Mart. & 3 & 3,947 & 0,32 & 5,26 & 0,34 & 0,011 & 0,06 & 0,24 \\
\hline Curatella americana $\mathrm{L}$. & 1 & 1,316 & 0,11 & 5,26 & 0,34 & 0,047 & 0,27 & 0,24 \\
\hline Inderterminada & 2 & 2,632 & 0,21 & 5,26 & 0,34 & 0,021 & 0,12 & 0,23 \\
\hline Parkia pendula (Willd.) Benth. ex Walp. & 1 & 1,316 & 0,11 & 5,26 & 0,34 & 0,035 & 0,2 & 0,22 \\
\hline Pisonia sp & 1 & 1,316 & 0,11 & 5,26 & 0,34 & 0,007 & 0,04 & 0,16 \\
\hline Diplopterys A.Juss. & 1 & 1,316 & 0,11 & 5,26 & 0,34 & 0,005 & 0,03 & 0,16 \\
\hline Aspidosperma pyrifolium Mart. & 1 & 1,316 & 0,11 & 5,26 & 0,34 & 0,003 & 0,02 & 0,15 \\
\hline Croton blanchetianus Baill. & 1 & 1,316 & 0,11 & 5,26 & 0,34 & 0,002 & 0,01 & 0,15 \\
\hline Aspidosperma cuspa (Kunth) S.F.Blake ex Pittier & 1 & 1,316 & 0,11 & 5,26 & 0,34 & 0,002 & 0,01 & 0,15 \\
\hline Inderterminada & 1 & 1,316 & 0,11 & 5,26 & 0,34 & 0,001 & 0,01 & 0,15 \\
\hline Total & 945 & 1244,737 & 100 & 1531,58 & 100 & 17,219 & 100 & 100 \\
\hline
\end{tabular}

As espécies Curatella americana, Parkia pendula , Pisonia sp, Diplopterys , Aspidosperma pyrifolium ., Croton blanchetianus, Aspidosperma cuspa foram as que obtiveram os menores valores para densidade, dominância e frequência (Tabela 2). Dessa forma, baixa presença de espécies pioneiras evidencia ainda mais que a área encontra-se em bom estado de conservação.

Quanto ao índice de diversidade de ShannonWeaver (H') observado para a área de estudo, este corresponde a 3,08 nats. ind $^{-1}$. Com relação ao índice de Dominância de Simpson $(\mathrm{C})$ que ocorre na escala de 0 a 1 , o valor observado neste estudo foi de 0,93. O índice de equabilidade de Pielou (J') é derivado do índice de diversidade de Shannon e permite representar a uniformidade da distribuição dos indivíduos entre as espécies existentes, os seus valores variam de 0 a 1 , quanto mais próximo de 1 mais abundante é a área, o valor observado neste trabalho foi de 0,79 .
Este trabalho apresentou valores superiores, porém bem próximo aos estudos realizados por Leite et al. (2015), que estudaram a vegetação lenhosa da Caatinga em Teixeira, PB, e encontraram um valor de 3,06 nats.ind ${ }^{-1}$. Os valores também foram superiores aos encontrados por Leitão et al. (2014), que pesquisaram a florística e estrutura de uma área transicional Caatinga Mata Atlântica, e verificaram 3,05 nats.ind ${ }^{-1}$. Alves et al. (2013), analisaram um componente florestal de uma área de Caatinga no município de Bom Jesus, no estado do Piauí e constataram 2,96 nats.ind ${ }^{-1}$, bem como o valor encontrado por Souza et al 2017, que analisaram a composição e estrutura da vegetação de caatinga no sul do Piauí, que constataram 2,7 nats.ind ${ }^{-1}$.

\section{Estoque Florestal}

Para a distribuição do número de indivíduos por classes de altura, a maior concentração ocorreu na classe intermediária $(3,88 \leq \mathrm{H}<11,42)$ (Figura 3).

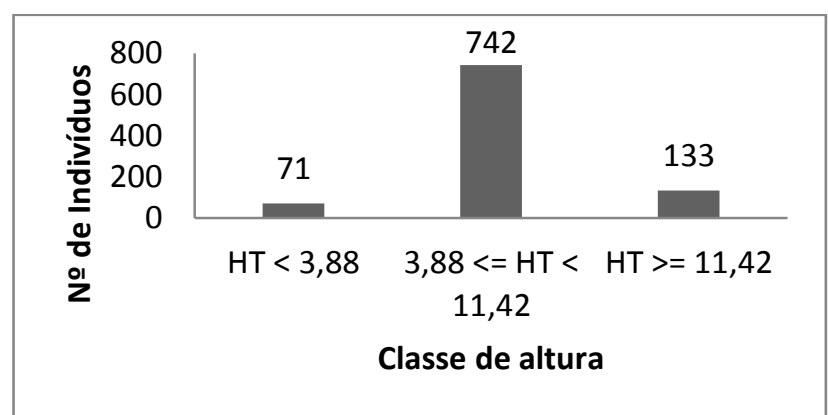

Figura 1. Número de indivíduos por classe de altura por hectare em área na UPF Umbaúba II, São Francisco do Piauí.

A altura média estimada foi de 6,7 metros, já a altura máxima foi 13 metros para os indivíduos das espécies Annona leptopetala (R.E.Fr.) H Rainer., Aspidosperma parvifolium A.DC., Aspidosperma 
multiflorum A.DC, Terminalia fagifolia Mart, Machaerium acutifolium Vogel, Poincianella pyramidalis (Tul.) L.P.Queiroz, Senna acuruensis (Benth.) H.S.Irwin \& Barneby, Andira fraxinifolia Benth, Hymenaea courbaril L. e Brosimum Sw.

Resultado semelhante a do presente estudo foi observado por Souza, et al. (2017), que estudando a estrutura da vegetação no sul do Piauí encontrou uma altura média de 3,7 m. Já Alves et al. (2013), analisando a estrutura vegetacional em uma área de Caatinga no município de Bom Jesus, Piauí obteve o valor de 3,44. Santos et al, (2017) em uma análise florística-fitossociológica e potencial madeireiro de uma área de caatinga submetida a Manejo Florestal, em Desterro, Paraíba diagnosticaram um valor de $3,8 \mathrm{~m}$. Alves et al. (2013) ressalta que o grau de perturbação encontrado em algumas área de estudos pode ser um fator que influencia diretamente a baixa altura média das árvores encontradas nessas comunidades. Desta forma, o valor superior aqui encontrado permite inferir que as condições naturais em que se encontra o fragmento estudado são mais preservadas do que as áreas dos levantamentos aqui comparados, outro sim, é o porte considerado alto, uma vez que, apresentou superioridade quando comparado com vários estudos realizados no Bioma Caatinga.
Considerando o fator de forma e fator de empilhamento, as áreas apresentaram uma estimativa média para o volume empilhado de aproximadamente 466,76 st.ha $^{-1}$, considerando todos os indivíduos florestais amostrados 455,91 st.ha ${ }^{-1} \mathrm{e}$, também considerando as restrições de corte (Tabela 4).

O valor calculado para volume real da área foi de $112,97 \mathrm{~m}^{3} \cdot \mathrm{ha}^{-1}$, resultados inferiores foram observados por Silva (2005), que encontrou 32,73 $\mathrm{m}^{3}$. ha ${ }^{-1}$, na Fazenda Oriente no Município de Condado-PB, microrregião de Sousa-PB, Sousa et al. O número médio de indivíduos nas classes entre de $2 \mid-7 \mathrm{~cm}$ e de $7 \mid-12 \mathrm{~cm}$ indica que essas classes possuem densidade alta e com predominância de indivíduos com diâmetro pequeno. Os indivíduos estão distribuídos de forma que $50,15 \%$ da população têm entre 2 e $7 \mathrm{~cm}$ de e $26,70 \%$ entre 7 a $12 \mathrm{~cm}$, de totalizando $76,85 \%$ da população com diâmetros entre 2 a $12 \mathrm{~cm}$ (Tabela 4).

A predominância de indivíduos nas primeiras classes de diâmetro, apresentando padrão de $\mathrm{J}$ invertido, enfatiza o padrão normal para floresta inequiâneas (ALVES et al., 2013; LIMA; COELHO, 2015; GUEDES et al., 2012).

Tabela 4. Distribuição da Área Basal (AB), Volume Real (VR) e Volume Empilhado (VE) por classe de diâmetro em área de Caatinga na UPF Umbaúba II, São Francisco, Paraíba, Piauí.

\begin{tabular}{ccccc}
\hline & & & & \\
\hline Classe & $\mathbf{N}$ & $\mathbf{A B}$ & Vol. Real (m) $/ \mathbf{h a})$ & Vol. emp (st/ha) \\
$\mathbf{2} \mid-\mathbf{7}$ & 650 & 1,203 & 6,024 & 24,887 \\
$\mathbf{7} \mid-\mathbf{1 2}$ & 346 & 2,329 & 15,035 & 62,116 \\
$\mathbf{1 2} \mid-\mathbf{1 7}$ & 159 & 2,501 & 19,690 & 81,352 \\
$\mathbf{1 7} \mid-\mathbf{2 2}$ & 72 & 2,187 & 20,548 & 84,895 \\
$\mathbf{2 2} \mid-\mathbf{2 7}$ & 27 & 1,271 & 13,049 & 53,911 \\
$\mathbf{2 7} \mid-\mathbf{3 2}$ & 25 & 1,615 & 16,770 & 69,285 \\
$\mathbf{3 2} \mid-\mathbf{3 7}$ & 12 & 1,128 & 11,870 & 49,043 \\
$\mathbf{3 7} \mid-\mathbf{4 2}$ & 2 & 0,222 & 2,593 & 10,712 \\
$\mathbf{4 2} \mid-\mathbf{4 7}$ & 1 & 0,147 & 1,722 & 7,115 \\
$\mathbf{4 7} \mid-\mathbf{5 2}$ & 0 & 0,000 & 0,000 & 0,000 \\
$\mathbf{5 2} \mid-\mathbf{5 7}$ & 2 & 0,485 & 5,674 & 23,442 \\
\hline Total & $\mathbf{1 2 9 6}$ & $\mathbf{1 3 , 0 8 8}$ & $\mathbf{1 1 2 , 9 7 4}$ & $\mathbf{4 6 6 , 7 6}$ \\
\hline
\end{tabular}

$\mathrm{Na}$ figura 2, observa-se que a distribuição percentual do volume empilhado por classes diamétricas possui uma tendência do modelo de distribuição de caracterização típica do cenário de florestas naturais. Por outro lado, a área destinada ao Manejo Florestal apresentou um potencial maior, 
para destinação de uso mais nobre como estacas, moirões e construções rurais no geral, o que termina por influenciando para um maior retorno econômico para o proprietário.

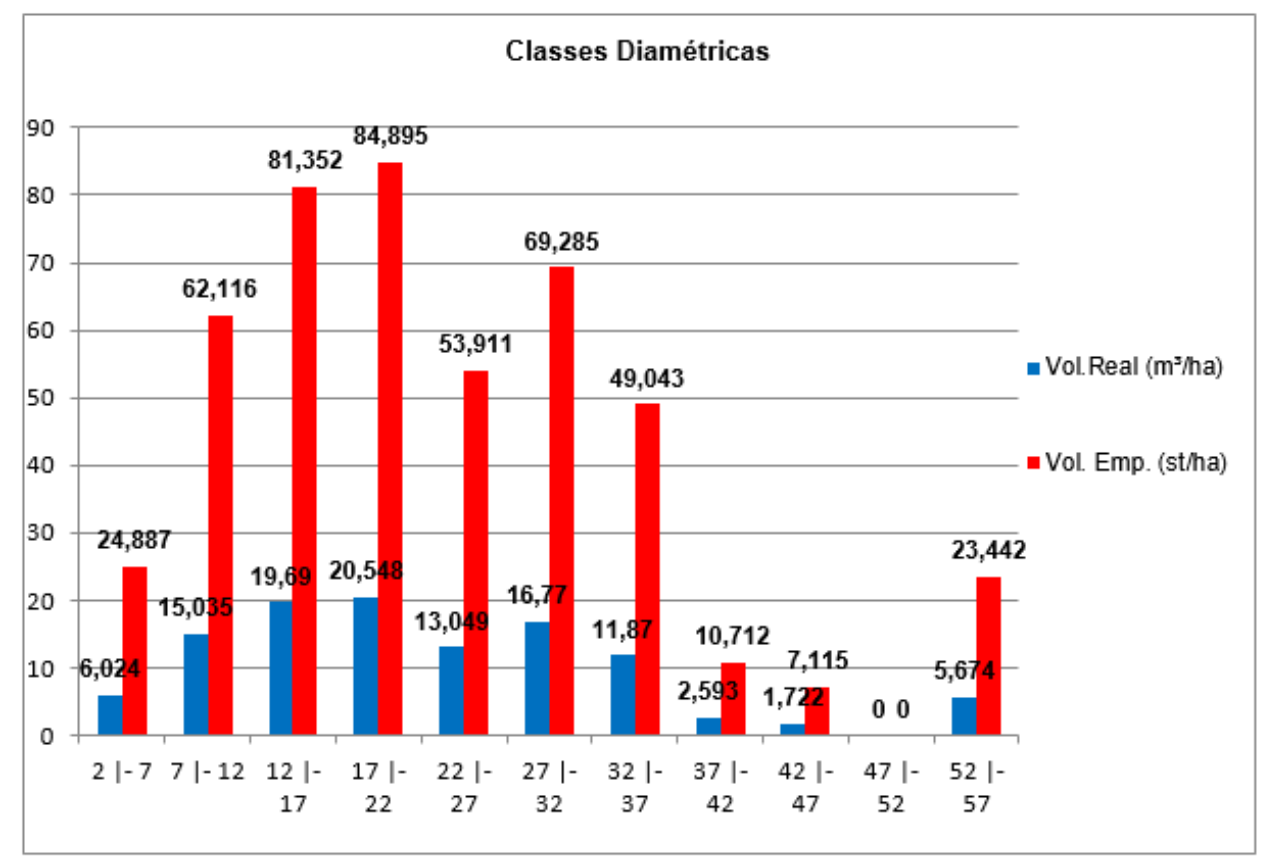

Figura 2. Percentual do Volume real por amostra (Vol real) e Volume empilhado por hectare (Vol emp) por classes diamétricas da UPF Umbauba II.

Atualmente, não se dispõe de nenhuma informação sobre o Incremento Médio Anual (IMA) na área específica e consequentemente o ciclo de corte. Este IMA é relativamente baixo considerando as condições edafo-climáticas da região. Será preciso monitorar a evolução do estoque florestal após 5

\section{CONCLUSÃO}

As famílias botânicas que apresentaram maior número de indivíduos foram Fabaceae, Apocynaceae e Combretaceae. As espécies com maior número de indivíduos e maiores valores de importância da área foram Terminalia fagifolia, Combretum glaucocarpum., Xylopia sericea, Aspidosperma discolor, Caryocar sp.

Para todo o efeito de comparação, a diversidade de espécies no fragmento estudado foi considerada elevada para o padrão normal em áreas de Caatinga, haja vista que quando se compara com outros estudos, os valores são relativamente inferiores ao encontrado no presente estudo.

O componente florestal estudados constitui em uma área de transição vegetacional Caatinga/ Cerrado, uma vez que, na mesma, há ocorrência de espécies de ambos os ambientes, com maior predominância das espécies pertencentes ao bioma Caatinga.

A produção madeireira mensurada na área foi de $112,97 \mathrm{~m}^{3} \cdot \mathrm{ha}^{-1}$, considerado elevado para região quando comparada com outras áreas de Caatinga no estado do Piauí. anos para avaliar o crescimento da vegetação. Logo, é esperado um ciclo de corte de 15 anos na área, que, permitirá, no futuro, a obtenção de uma produção maior do que a encontrada e, além disto, uma recuperação significativa da Caatinga.

\section{REFERÊNCIAS}

AGUIAR, R. de B; GOMES, J. R. de C. Projeto cadastro de fontes de abastecimento por água subterrânea, Piauí: Diagnóstico do município de Bom Jesus do PiauíCPRM-Fortaleza, 2004.

ANGIOSPERM PHYLOGENY GROUP (APG II). 2003. An update of the Angiosperm Phylogeny Group classification for the orders and families of flowering plants: APG II. Botanical Journal of the Linnean Society 141: 399-436.

ALVES, A. R.; RIBEIRO, I. B.; SOUSA, J. R. L.; BARROS, S. S.; SOUSA, P. R. Análise da estrutura vegetacional em uma área de caatinga no município de Bom Jesus, Piauí. Revista Caatinga, Mossoró, v. 26, n. 4, p. 99-106, 2013.

ANDRADE, L.A.; PEREIRA, I.M.; LEITE, U.T.; BARBOSA, M.R.V. Análise de cobertura de duas fitofisionomias de caatinga, com diferentes históricos de uso, no município de São João do Cariri, Estado da Paraíba. Revista Cerne, Lavras (MG) v. 11, n. 3, p.253262, 2005. 
BULHÕES, A. A.; CHAVES, A. D. C. G.; ALMEIDA, R. R. P.; RAMOS, I. A. N.; SILVA, R. A.; ANDRADE, A. B. A.; SILVA, F. T. Levantamento Florístico e Fitossociológico das Espécies Arbóreas do Bioma Caatinga realizado na Fazenda Várzea da Fé no Município de Pombal-PB. Informativo Técnico do Semiárido, Mossoró, v.9, n.1, p.51-56, 2015.

CHAVES, A. D. C. G.; SANTOS, R. M. S.; SANTOS, J. O.; FERNANDES, A. A.; MARACAJÁ, P. B. A importância dos levantamentos florístico e fitossociológico para a conservação e preservação das florestas. Agropecuária Científica no Semiárido, Campina Grande, v.9, n.2, p. 43-48, 2013.

CALIXTO JÚNIOR, J. T.; DRUMOND, M. A. Estudo comparativo da estrutura fitossociológica de dois fragmentos de Caatinga em níveis diferentes de conservação. Pesquisa Florestal Brasileira, Colombo, v. 34, n. 80, p. 345-355, 2014.

DIAS, C. T. de V. KIILL, L. H. P. Levantamento florístico da reserva legal do Projeto Salitre, JuazeiroBA. Petrolina, PE: Embrapa Semiárido, 2008.

FERNANDES, J. D. MEDEIROS, A. J. D. de Desertificação no nordeste: uma aproximação sobre o fenômeno do Rio Grande do Norte. Holos, v.3, ano 25. Rio Grande do Norte, 2009.

FORZZA, R. C. et al. (coords). Lista de Espécies da Flora do Brasil. Rio de Janeiro: Jardim Botânico, 2012.

DRUMOND, M. A.; KILL, L. H. P.; LIMA, P. C. F.; OLIVEIRA, M. C. de; OLIVEIRA, V. R. de; ALBUQUERQUE, S. G. de; NASCIMENTO, C. E. de S.; CAVALCANTI, J. Estratégias para o Uso Sustentável da Biodiversidade da Caatinga. In: Avaliação e identificações de ações prioritárias para a conservação, utilização sustentável e repartição dos benefícios da biodiversidade do bioma caatinga. In: SEMINÁRIO BIODIVERSIDADE DA CAATINGA, I. Anais... Petrolina: Embrapa Semiárido, 2000.

FELFILI, J. M.; REZENDE, R. P. Conceitos e métodos em fitossociologia. Universidade de Brasilia, Brasilia, 2003. p.44-53.

GAMARRA-ROJAS, C. F. L. et al. (Eds.). Banco de Dados de Plantas do Nordeste. Checklist das Plantas do Nordeste (versão 1.5). Disponível em: <http://www.cnip.org.br/bdpn/>. Acesso em: 30/Ago./2017.

GARIGLIO, A. M. A rede de manejo florestal da Caatinga. In: GARIGLIO, M. A.; SAMPAIO, E. V. S.B.; CESTARO, L. A.; KAGEYAMA, P. Y. (orgs). Uso sustentável e conservação dos recursos florestais da Caatinga. Brasília, Serviço Florestal Brasileiro, 2010. Cap.4, p. 199-204.
GUEDES, R. S.; ZANELLA, F. C. V.; JÚNIOR, J. E. V. C.; SANTANA, G. M.; SILVA, J. A. Caracterização florístico-fitossociológica do componente lenhoso de um trecho de caatinga no semiárido paraibano. Revista Caatinga, Mossoró, v.25, n.2, p.99-108, 2012.

LEITE, J.A.N.; ARAÚJO, L.V.C.; ARRIEL, E.F.; CHAVES, L.F.C.; NÓBREGA, A.M.F. Análise quantitativa da vegetação lenhosa da Caatinga em Teixeira, PB. Pesquisa Florestal Brasileira, Colombo, v.35, n.82, p.89-100, 2015. http://dx.doi.org/ 10.4336/2015.pfb.35.82.584.

LEITÃO, A. C.; VASCONCELOS, W. A.; CAVALCANTE, A. M. B.; TINÔCO, L. B. M.; FRAGA, V. S. Florística e estrutura de um ambiente transicional caatinga-mata atlântica. Revista Caatinga, Mossoró, v.27, n.3, p.200-210, 2014.

LIMA, B. G. Caatinga: espécies lenhosas e herbáceas. Mossoró: Ed. UFERSA, 2012. 316 p.

LORENZI, H. Árvores brasileiras: manual de identificação e cultivo de plantas arbóreas nativas do Brasil. 4 ed. Nova Odessa, São Paulo: Instituo Plantarum, 2002. 368 p. v.1.

MAIA, G. N. Caatinga: árvores e arbustos e suas utilidades. 2. ed. Fortaleza: Printcolor Gráfica e Editora, 2012. $413 \mathrm{p}$.

LIMA, B. G.; COELHO, M. F. B. Estrutura do componentearbustivo-arbóreo de um remanescente de caatinga no estado do Ceará, Brasil. Cerne, v. 21, n. 4, p. 665-672, 2015.

LORENZI, H. Árvores brasileiras: manual de identificação e cultivo de plantas arbóreas nativas do Brasil. 4 ed. Nova Odessa, São Paulo: Instituo Plantarum, 2002. 368 p. v.1.

MAIA, G. N. Caatinga: árvores e arbustos e suas utilidades. 2. ed. Fortaleza: Printcolor Gráfica e Editora, 2012. 413 p.

PRADO, D. E. As caatingas da América do Sul. In: Ecologia e Conservação da Caatinga. Recife: Ed. Universitária da UFPE, 2003. 822 p.

RODAL, M. J. N. Manual sobre métodos de estudos florístico e fitossociológicos - ecossistema caatinga. Sociedade botânica do Brasil. Janeiro: 1992. 23 p.

SAMPAIO, E. V. S. B.; PAREYN, F. G. C.; FIGUEIRÔA, J. M.; SANTOS JUNIOR, A. G. S. (Eds.). Espécies da flora nordestina de importância econômica potencial. Recife: Associação Plantas do Nordeste, 2005. 331p. 
SANTOS, W. S.; HENRIQUES, I.G.N.; SANTOS, W. S.; RAMOS, G.G.; VASCONCELOS, G. S.

VASCONCELOS, A.D.M. Análise florísticafitossociológica e potencial madeireiro em área de caatinga submetida a manejo florestal. Agropecuária Científica no Semiárido, Patos-PB, v.13, n.3, p.203-211, 2017.

SILVA, J. S.; SALES, M. F. de; GOMES, A. P. de S.; CARNEIRO-TORRES, D. S. Sinopse das espécies de Croton L. (Euphorbiaceae) no estado de Pernambuco, Brasil. Acta Botânica Brasílica, São Paulo, v.24, n.2, p.441-453, 2010.

SILVA, J. S.; SALES, M. F. de; GOMES, A. P. de S.; CARNEIRO-TORRES, D. S. Sinopse das espécies de Croton L. (Euphorbiaceae) no estado de Pernambuco, Brasil. Acta Botânica Brasílica, São Paulo, v.24, n.2, p.441-453, 2010.

SILVA, J. A. Fitossociologia e relações alométricas em caatinga nos estados da Paraíba e Rio Grande do Norte. 2005. 81 f. Tese (Doutorado em Engenharia Florestal) - Universidade Federal de Viçosa, Viçosa, MG, 2005.
SIQUEIRA FILHO, J. A.; SANTOS, A. P. B.; NASCIMENTO, M. F. S.; ESPIRITO SANTO, F. S. Guia de Campo de Árvores da Caatinga. Petrolina: Editora e Gráfica Franciscana Ltda., 2009. 64 p.

SOUZA, M. P.; COUTINHO, J.M.C.P.; SILVA, L..S.; AMORIM, F.S.; ALVES, A.R. Composição e estrutura da vegetação de caatinga no sul do Piauí, Brasil. Revista Verde de Agroecologia e Desenvolvimento Sustentável V.12, No 2, p. 210-217, 2017.

SOUZA, P. F. Análise da vegetação de um fragmento de caatinga na microbacia do açude jatobá. Patos-PB, 2009. 51 p. Trabalho de conclusão de curso (Engenharia Florestal) - Universidade Federal de Campina Grande, Patos, PB, 2009.

ZAKIA, M. J. B; PAREYN, F. G. C.; RIEGELHAUPT, E. Equações de Peso e Volume para Oito Espécies Lenhosas Nativas do Seridó-RN. In: Plano de Manejo Florestal para a Região do Seridó-RN. cap. 4, v. 1, Natal: Projeto PNUD/FAO/IBAMA/BRA/87/007, 1988. 